\title{
Overview of Recent Pedestal Studies at ASDEX Upgrade
}

E. Wolfrum ${ }^{1}$, E. Viezzer ${ }^{1}$, A. Burckhart ${ }^{1}$, M.G. Dunne ${ }^{1}$, P.A. Schneider ${ }^{1}$, M. Willensdorfer ${ }^{1}$, E. Fable $^{1}$, R. Fischer ${ }^{1}$, D. Hatch ${ }^{3}$, F. Jenko ${ }^{1}$, B. Kurzan ${ }^{1}$, P. Manz ${ }^{2}$, S.K. Rathgeber ${ }^{1}$ and the ASDEX Upgrade Team

${ }^{1}$ Max-Planck-Institut für Plasmaphysik, Garching, Germany

${ }^{2}$ Physik-Department E28, Technische Universität München, Garching, Germany

${ }^{3}$ Institute for Fusion Studies, University of Texas at Austin, Austin, Texas, USA

E-mail contact of main author: e.wolfrum@ipp.mpg.de

\begin{abstract}
New or upgraded diagnostics of the edge transport barrier allow investigations of the dominant transport mechanisms in the pedestal. The density build-up after the L-H transition can be explained with a mainly diffusive edge transport barrier. A small inward convection term improves the agreement between modelling and experiment, but its existence cannot be confirmed due to the uncertainty in the neutral sources. Measurements of the impurity ion flow asymmetry as well as the edge current density are in agreement with neoclassical modelling. The inter-ELM pedestal recovery was traced with ideal peeling-ballooning modelling, which shows that the stability boundary moves closer to the operational point as the pedestal becomes wider. Gyrokinetic modelling of the different phases reveal that density gradient driven trapped electron modes are dominant during the early recovery, while electron temperature gradient modes or kinetic ballooning modes determine the temperature gradient in the final phase. Micro tearing modes are modelled and also experimentally determined at the top of the pedestal. Non linear coupling between modes could explain the failure of ideal linear MHD modelling.
\end{abstract}




\section{Introduction}

The edge transport barrier (ETB) is a characteristic of the high confinement mode (H-mode) of tokamaks. It is a narrow region of reduced radial transport, in which steep gradients in both density and temperature are observed, forming a pedestal for the core profiles.

Extensive studies have shown that the pedestal radial electric field $\left(E_{\mathrm{r}}\right)$ profile in H-mode [1] and asymmetric density and flow profiles of impurity ions are consistent with neoclassical predictions [2]. While the ions set the background flow profile in the pedestal and their transport properties can be described by neoclassical modelling, the mechanisms which determine the electron density and temperature profiles are more varied, as electron heat and particle transport are governed by turbulence. The current emphasis of research concentrates on the determination of the driving force as well as the characteristics of the dominant transport mechanism. The understanding of the types of instabilities and the transport they create in the core plasma has been greatly advanced in recent years $[3,4]$. Although it has been shown that peeling ballooning (PB) theory in combination with the assumption that the pedestal width is determined by kinetic ballooning modes (KBM) [5, 6] describes the pedestal top in a wide range of pedestal pressures [7], the detailed processes which determine the ion and electron heat transport as well as particle transport are not yet clearly identified. Improvements in numerical methods and further developments of gyrokinetic theory now facilitate studies in the edge transport barrier [8, 9].

An essential ingredient for such studies is the development of edge plasma diagnostics with high spatial and temporal resolution, as well as the completeness of a dataset necessary for modelling. At ASDEX Upgrade, the available set of edge diagnostics consisting of Thomson scattering (TS) [10], Lithium beam (LiB) [11], electron cyclotron emission (ECE), ECE imaging (ECEI) [12] and edge charge exchange recombination spectroscopy (CXRS) [13] has been improved and extended by electron cyclotron forward modelling (ECFM) [14], additional views of CXRS for $E_{r}$ measurements [15] and the determination of the edge current density from a combination of magnetic measurements, edge pressure profiles and scrape-off layer (SOL) current measurements [16]. The combinations of these diagnostics deliver a set of profiles consisting of electron density $\left(\mathrm{n}_{\mathrm{e}}\right)$, electron temperature $\left(T_{e}\right)$, ion temperature $\left(T_{i}\right)$, radial electric field $\left(E_{r}\right)$ and edge current density $(j)$, with temporal resolutions down to $1 \mu$ s (ECE) to 2 ms (CXRS), which is high enough to study the build-up of the edge transport barrier after an L-H transition or after the collapse due to an edge localised mode (ELM). 
This paper reviews current pedestal studies at ASDEX Upgrade and is structured as follows: After an overview over the improvements in diagnostic capability in the pedestal region, which is given in section 2, several studies related to pedestal transport and pedestal stability are described. Section 3 summarizes the findings of particle transport modelling immediately after the L-H transition. In section 4 it is demonstrated that ion flows in the pedestal can be described by neoclassical theory. The remainder of the paper is dedicated to studies of the inter-ELM phase, which consists of several phases with distinct behaviour of density and temperature profile recovery. In section 5 the operational points of these phases are studied in the frame of ideal peeling-ballooning theory, while in section 6 they are analysed via gyrokinetic modelling.

\section{New or improved diagnostics}

An essential prerequisite for pedestal studies are measurements of $n_{e}$ and $T_{e}$ profiles with high spatial and temporal resolution. While the TS diagnostic provides profiles of $n_{e}$ and $T_{e}$ at the same position and is used for the relative alignment of these profiles, ECE and LiB diagnostics deliver profiles with higher temporal resolution (1 MHz and $200 \mathrm{kHz}$, respectively). This excellent temporal resolution of $\mathrm{LiB} \mathrm{n}_{\mathrm{e}}$ profiles was accomplished by the installation of a new optical head with high photon collection efficiency [17]. Integrated data analysis (IDA) within a Bayesian framework interprets these data in combination with line integrated $n_{e}$ measurements [18] to provide the $n_{e}$ and $T_{e}$ profiles [19] used in pedestal studies. A new 1D model of electron cyclotron radiation transport has been developed, which takes into account relativistic effects as well as the optical depth of the local plasma [14], and allows $T_{e}$ gradients in the ETB to be determined accurately. A set of reflectometry diagnostics are in operation at ASDEX Upgrade, which however were not used in the investigations presented in this work. Figure 1 shows a complete set of pedestal profiles, in which the data are ELM synchronized in an interval of one second, which also contains a radial sweep of the plasma to improve the radial resolution. Only data in the time window $4 \mathrm{~ms}$ to $1 \mathrm{~ms}$ before an ELM are taken. The separatrix location is taken from the equilibrium reconstruction, while the relative $T_{e}$ profile alignment is determined by the two-point model [20] and for typical H-modes $T_{e}$ at the separatrix is close to $100 \mathrm{eV}$. In figure $1 \mathrm{a} \mathrm{T}_{\mathrm{e}}$ profiles from TS is shown, the radiation temperatures from the ECE measurements and the $T_{e}$ profile derived from the ECFM method, clearly demonstrating that ECE radiation temperatures in the 
pedestal are affected by the so-called 'shine through', while the ECFM $\mathrm{T}_{\mathrm{e}}$ profile gives steeper profiles, in good agreement with the TS data. Figure $1 b$ shows $n_{e}$ profiles measured by TS and LiB. Relative alignments between the diagnostics are within the respective experimental uncertainties (5 $\mathrm{mm}$ ), and are carried out in order to guarantee an optimized alignment between $T_{e}$ from ECE and $n_{e}$ from LiB. A new poloidal CXRS [15] system has been added to the already existing toroidal CXRS system, measuring both $T_{i}$ and rotation velocities. Figure $1 d$ shows the edge $T_{i}$ profiles from both systems (ELM synchronized) as well as $T_{i}$ from the core CXRS system [21] (not ELM synchronized), measured from charge exchange of the $\mathrm{B}^{5+}$ impurity ion. The toroidal and poloidal rotation velocity profiles are plotted in $1 \mathrm{e}$, and $1 \mathrm{f}$ shows the profile of $\mathrm{E}_{\mathrm{r}}$ as derived from the radial force balance equation of the measured impurity ion. The $E_{r}$ profile can be evaluated directly from the CXRS measurements since all quantities of the observed ion species, which are needed for the calculation of $E_{r}$, are determined from the measured spectra. Two additional edge CXRS systems were taken into operation in ASDEX Upgrade on the high field side [22], which are used to determine the poloidal asymmetries discussed in section 4.

In order to test the peeling-ballooning model [23], profiles of both the pressure gradient, which is determined experimentally via the diagnostics and methods already described, and of the edge current density are required. The edge current density has typically been treated either as an unknown quantity, or has been assumed to be described by neoclassical theory throughout the ELM cycle. A new method has recently been deployed at AUG which makes use of an equilibrium solver in combination with the extensive suite of edge kinetic, external magnetic, and SOL current measurements available [16]. The SOL currents are determined experimentally via shunt current measurements at the divertor tiles, the edge kinetic profiles are used as a constraint to the interpretive equilibrium reconstruction, which produces a best fit to all magnetic data. Via this method, the edge current density profile shape and magnitude could be recovered at a high temporal resolution and on a spatial scale which allows detailed analysis in the pedestal region. Figure 1c shows the edge current density profile for the pre-ELM interval, which uses also ELM synchronized magnetic data and SOL current measurements. The high temporal resolution of $n_{e}$ and $T_{e}$ measurements via LiB and ECE, respectively, allows the studies of the density build up after the L$\mathrm{H}$ transition as described in the next section. The extended set of diagnostics on ion profiles is a prerequisite for the studies presented in section 4 and the complete set of data was used for an ELM cycle analysis as shown in sections 5 and 6 . 



Figure 1: Edge profiles of a) $T_{e}$, b) $n_{e}$, c) jpeak_edge, d) $T_{i}$, e) $v_{\text {tor }}$ and $v_{\text {pol }}$, and f) $E_{r}$. All data are ELM synchronized and only data taken in an interval of -4 to -1 ms before an ELM (except core CXRS data).

\section{Density build-up after the L-H transition}

Edge transport decreases with the transition from the low confinement mode (L-mode) to the high confinement mode (H-mode) of tokamak plasmas. In the fluid picture modelling relies on the description of particle transport via diffusion and convection. Especially the existence of a convective term would relax the fuelling worries in ITER [24]. The highly temporally resolved $n_{e}$ profiles after an L-H transition show a characteristic density build-up. This density build-up was modelled with the transport code ASTRA in a predictive-iterative way [25]. The convective velocity, diffusion coefficient and the particle source profiles were parameterized and their parameters were varied until the best match of the modelling to the temporal evolution of the density measurements was found. The density build-up can be reproduced by assuming a diffusive ETB only, with a reduced diffusion coefficient at the edge with respect to the core values. When an edge pinch is added to this fully diffusive ETB, slightly better agreement between experiment and modelling is observed. However, due to the uncertainty in the neutral particle sources, the existence of a pinch cannot be confirmed by this analysis. The best agreement was found with a diffusion coefficient of $0.031 \mathrm{~m}^{2} \mathrm{~s}^{-1}$ and a convective velocity of $-0.5 \mathrm{~ms}^{-1}$ in the pedestal [25]. In order to determine the effect of $\mathrm{T}_{\mathrm{e}}$ on edge particle transport in the pedestal, the density build-up after the L- 
$\mathrm{H}$ transition was analyzed at different heating powers. Electron cyclotron resonance heating was used to vary the pedestal temperature during the density evolution between subsequent H-mode phases. Although the pedestal $\mathrm{T}_{\mathrm{e}}$ and its gradients could be varied by a factor of two, no change in the edge density evolution was observed. Again ASTRA was used to interpret the measurements and derive pedestal particle transport coefficients. Thermo-diffusion seems to play a minor role in the pedestal [26].

\section{Consistency between ion measurements and neoclassical theory}

A detailed study combining the $\mathrm{E}_{\mathrm{r}}$ data with the ion and electron profile measurements revealed that in the fully developed $\mathrm{H}$-mode the edge $\mathrm{E}_{\mathrm{r}}$ and the main ion pressure gradient term, $\nabla \mathrm{p}_{\mathrm{i}} /\left(\mathrm{en}_{\mathrm{i}}\right)$, are identical within the experimental uncertainties. This relation is confirmed by direct measurements of the temperature, density and rotation profiles of the main ions in helium plasmas. The measurements demonstrate that the perpendicular main ion flow is close to zero in the pedestal and is consistent with neoclassical predictions [27].

New edge CXRS measurements at the high-field side (HFS) of ASDEX Upgrade [22] allow the poloidal flow structure in the ETB to be measured. The HFS and low field side (LFS) profiles are aligned relative to each other by assuming that $T_{i}$ is a flux function. After the alignment the following flow structure is observed in the fully developed H-mode: In the pedestal the poloidal impurity flow exhibits a strongly sheared rotation in the electron diamagnetic drift direction both at the LFS and HFS and the radial position of the minimum is approximately the same (see figure 2a). The HFS poloidal rotation is about a factor of 1.5-2 lower than at the LFS. The toroidal rotation is co-current at both the LFS and HFS and exhibits an asymmetric structure on the flux surfaces. At the LFS the toroidal rotation velocity has a minimum located around the pedestal top, while at the HFS the profile is reversed and exhibits a maximum at this position (see figure $2 \mathrm{~b}$ ). 


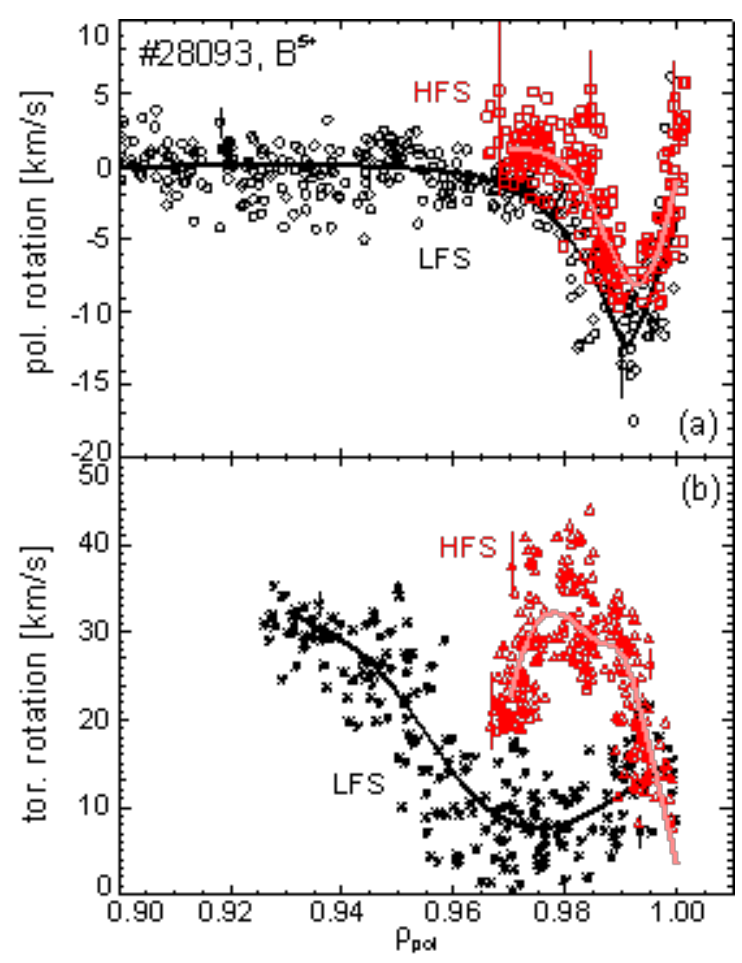

Figure 2: (a) Poloidal and (b) toroidal rotation at the HFS (red) and LFS (black) measured with CXRS on $\mathrm{B}^{5+}$. Only data taken during the $5 \mathrm{~ms}$ before an ELM are taken.

The toroidal impurity flow at the LFS increases towards the separatrix, while it decreases at the HFS. When using the assumption of constant $T_{i}$ on a flux surface the radial position of the minimum in the $E_{\mathrm{r}}$ profiles matches approximately (with differences in the location of $\sim \mathrm{mm}$ ) between the HFS and LFS [28]. Calculating the electrostatic potential profiles from the measured $E_{r}$ profiles indicates that the potential may be lower at the HFS compared to the LFS, however, taking the experimental uncertainties into account, the potential could also be a flux function. Following the postulate of divergence-free flows on a flux surface, both the toroidal flow asymmetry and the difference in magnitude of the poloidal flow can be explained by the presence of an in-out impurity density asymmetry, with an impurity accumulation at the HFS [29]. The measured flow structure has been compared to theoretical predictions based on the parallel force balance [30]. The in-out impurity asymmetry arises due to the interplay of many terms. At the plasma edge, friction between the main ions and impurities is the dominant driving force. Close to the separatrix, the poloidal centrifugal term which is usually neglected, gives additional contributions to the observed in-out asymmetries. A quantitative description of the observed flow structure is obtained when including a finite poloidal main ion flow of $\sim 2 \mathrm{~km} / \mathrm{s}$ in the simulation, which is consistent with standard 
neoclassical calculations using the NEOART code [31]. This demonstrates that despite the presence of a poloidally asymmetric impurity density profile, the impurity particle transport is neoclassical, consistent with previous studies [32].

\section{ELM cycle studies - Peeling-ballooning analysis}

It is a well-established observation on AUG that the temperature and density profiles recover at different rates after the ELM crash. Figures 3a,b show these different phases for the gradients of $T_{e}$ and $n_{e}$ in the ETB region. The pedestal recovery after the ELM crash (ii) is split into several different parts: rapid $\nabla n_{e}$ recovery while $\nabla T_{e}$ recovery stalls (iii), $\nabla T_{e}$ recovery continues while $\nabla n_{e}$ stays constant (iv), completion of $\nabla n_{e}$ and $\nabla T_{e}$ recovery (v) and saturated pre-ELM phase (vi and i) [33].
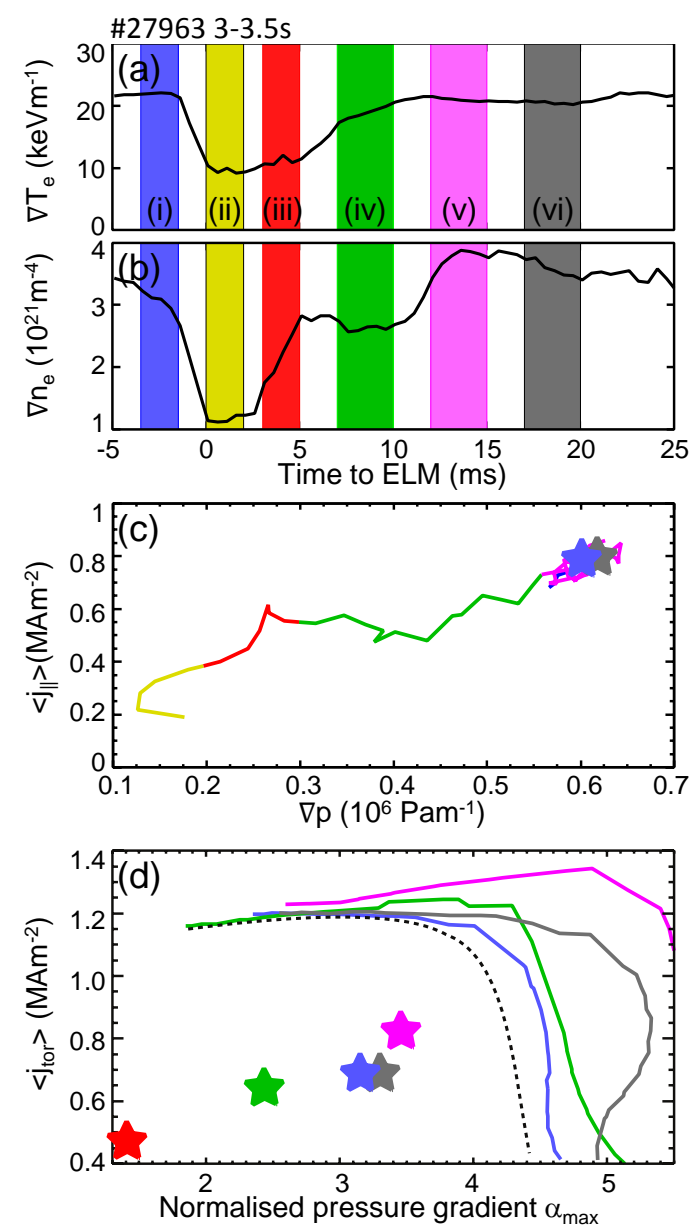
Figure 3: Recovery of the pedestal $\mathrm{T}_{\mathrm{e}}(\mathrm{a})$ and $\mathrm{n}_{\mathrm{e}}$ (b) gradients demonstrating the different phases. (c) Evolution of the edge current density vs. pressure gradient with the operational points of phases (i) and (vi) represented as stars. (d) Operational points (stars) and peeling ballooning stability boundaries for the phases denoted in (a) and (b) (colour coded) and at the ELM crash (dashed line).

The most successful model to explain the occurrence of ELMs is the peeling-ballooning (PB) model [34], which is also used as a constraint in the EPED model [35], a predictive model for the pedestal width and height. To test the PB model, high quality edge pressure and current density profiles are required. The determination of the edge current density profiles, described in Section 2, allowed a detailed comparison with neoclassical predictions to be performed. Similarly to the impurity density and flow profiles, discussed in Section 2, good agreement between experiment and theory was found [16]. Figure 3c shows the ELM resolved edge current density $\left.\left(<\mathbf{j}_{\|}\right\rangle\right)$versus the edge pressure gradient, with the different phases of the ELM cycle indicated. First, during the pre-ELM times, both pressure gradient and current density are constant. Following the ELM crash (yellow), the pressure gradient and current density begin to gradually recover as the density gradient builds up (red), followed by the temperature gradient (green). As soon as the pressure gradient saturates (purple), the edge current density also saturates. This indicates that there is indeed some mechanism clamping the pedestal at a critical gradient before the ELM crash and that some parameter other than the gradient or current density must be responsible for the destabilized pedestal. A detailed analysis of the profiles reveals that, although the gradient is clamped, the pedestal width continues to grow towards the end of the ELM cycle by about 15\%. Peeling-ballooning theory predicts that this should cause the stability boundary to move towards the operational point which is fixed in $\mathrm{j}-\alpha$ space, with $\alpha$ the normalized pressure gradient as in [36]. Using this data as input, the ideal linear MHD code suite ILSA/MISHKA [37] was used to determine the stability limit in the different phases of the ELM cycle. Figure 3d shows a stability diagram, with the operation points of the different phases as well as the respective stability boundaries. As expected, the operational point is far away from the stability limit in the early phases after the ELM crash. For phase (iii) only the operational point is plotted (red). Phase (iv) is characterized by an operational point (blue) which is far away from the corresponding stability boundary. Phase (v) shows the steepest pressure gradient together with the largest edge current (magenta), but the stability boundary has moved far away, most probably due to the combination of a still narrow pedestal and an increased core pressure. Later, the position of the operational points stay constant in $\mathrm{j}$ - $\alpha$ space while the stability limit moves 
closer until the ELM crash occurs. Due to the increasing width of the edge transport barrier region, more poloidal harmonics become unstable leading to the reduced $j-\alpha$ values of the stability boundary. Assuming a linear evolution of the stability boundary in time, an extrapolation of the boundary to the time of the ELM crash is depicted in figure 3d as dashed curve. However, the final ELM trigger condition cannot be determined by linear MHD stability alone, since the pre-ELM pressure gradient (blue star) is 30\% lower than the boundary, which is outside the experimental uncertainties of the measured edge kinetic profiles [38]. Several other cases were analysed in the same manner, and it was found that the operational point can lie either in the stable region or in the unstable region.

\section{ELM cycle studies - gyrokinetic analysis with GENE}

The same data have also been used as input for gyrokinetic simulations with GENE [39] in order to determine the dominant type of turbulence. This work is presented in detail in [9]. In the early phase of the ELM cycle density gradient driven trapped electron modes (TEM) are unstable and associated with a significant outward particle flux. These drift waves are susceptible to $\mathrm{E}_{\mathrm{r}} \mathrm{x} \mathrm{B}$ shear stabilization and are suppressed as the profiles recover during the ELM cycle, while kinetic ballooning modes (KBM) and microtearing modes (MTM) are present at larger scales. In the phase just before the ELM crash, the gyrokinetic analysis shows robustly unstable MTMs at the top of the pedestal, while the pedestal itself is slightly below the KBM limit.
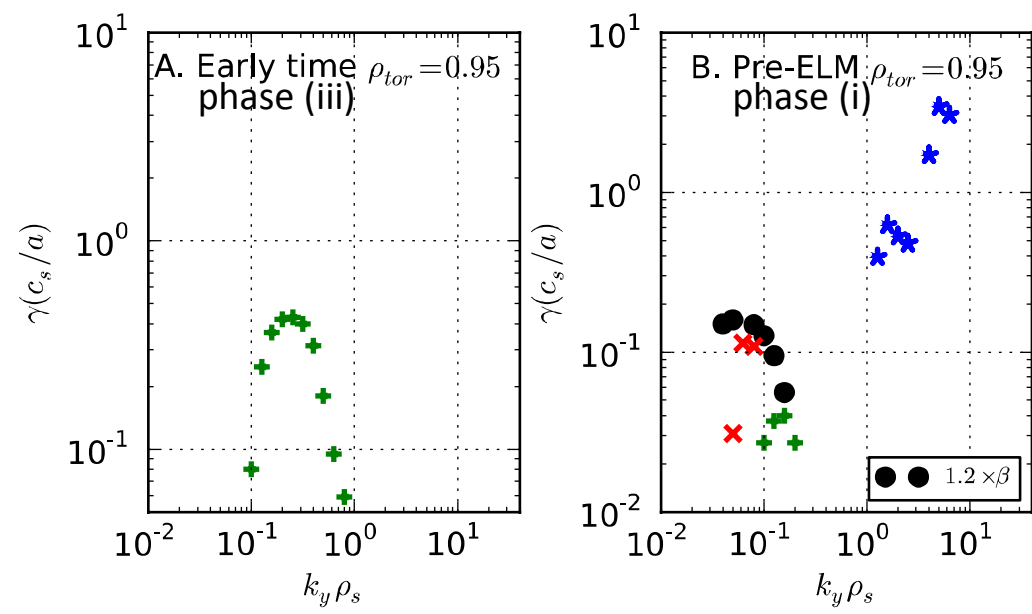
Figure 4: Growth rates in a) phase (iii) and b) phase (i) as determined by GENE modelling [39] in the steep gradient region of density gradient driven trapped electron modes (green crosses), ETG (blue stars), MTM (red crosses), KBM (black circles). Note that beta was increased by a factor of 1.2 to surpass the KBM threshold.

During the inter-ELM profile evolution, the density and temperature profiles separately undergo large changes while the pressure gradient is held fixed in the phases (v), (vi) and (i) and near the KBM limit, suggesting that KBMs constrain the profile evolution over the latter half of the ELM cycle. Figure 4 shows the growth rates of the main instabilities vs. $k_{y} \rho_{s}$ for the two phases: in the early phase (iii) and just before the ELM crash (phase (i)). The outward particle flux predicted by nonlinear simulations from the TEM dominated phase (iii), see figure $4 \mathrm{a}$, is in apparent contradiction with the increase in density in this early phase. This phase, however, is also marked by significant changes to the detachment state of the inner divertor and possible subsequent influences to the fuelling profile. Nonlinear simulations with varying density gradient scale lengths are used to estimate a particle diffusivity and pinch velocity. This analysis predicts values $(\mathrm{D}=$ $0.045 \mathrm{~m}^{2} / \mathrm{s}$ and pinch $\mathrm{v}_{\mathrm{p}}=-0.84 \mathrm{~m}^{2} / \mathrm{s}$ ) that are similar to those found in the transport analysis of the density build-up after the L-H transition described in section 3 [25]. Just before the ELM crash (phase (i), figure 4b), the pressure profile is near the KBM limit. Electron temperature gradient driven (ETG) modes are also unstable and could play a role in the edge transport barrier region [40]. Nonlinear ETG simulations demonstrate that the profiles lie at a nonlinear critical gradient; moderate changes to the profiles can produce an order of magnitude increase in heat flux. With such modifications, ETG transport can reach levels that would make a significant contribution to the pedestal electron heat flux. For the phase just before the ELM crash further experimental support is provided by the linear behavior of $\nabla \mathrm{T}_{\mathrm{e}} \mathrm{vs}$. $\mathrm{T}_{\mathrm{e}}$ in real space for a wide data base of pre-ELM pedestals [41]. The constancy of the temperature gradient length in real space for such a wide variation of shape, current, field and density hints towards a local mode (e.g. the KBM or ETG) rather than a global mode which limits the $T_{e}$ gradient. The existence of MTMs at the pedestal top is further supported by results of velocimetry analysis of ECEI data [42]. The spatial scales, parity and cross-phase between electron temperature and radial velocity fluctuations are evaluated by means of velocimetry of measured 2D electron temperature fluctuations. A comprehensive comparison with the properties of different instabilities points towards MTMs. Additionally, bi-spectral analysis of 
the same ECEI dataset provides evidence that the MTMs could couple to low-n ballooning modes, a fundamentally non-linear process which leads to the ELM crash.

\section{Conclusions}

The continuous improvement of the experimental diagnostics of the edge pedestal at ASDEX Upgrade has led to a new quality of analysis of the pedestal. Quantitative transport studies of the density build-up after the L-H transition have shown that if a pinch exists, it must be small, i.e. in the order of $-0.5 \mathrm{~ms}^{-1}$. Moreover, the particle transport is independent of the temperature and temperature gradient during the density build-up in these phases. A pinch of similar magnitude has been found to be driven by TEMs in the phase immediately after the ELM crash, in which the density gradient is building up while the temperature gradient is still low. While the ion flows as well as the bootstrap current can be described by neoclassical theory, the electrons are governed by a multitude of modes: at the top of the pedestal MTMs can be found, in the gradient region KBMs and/or ETG modes clamp the pre-ELM pedestal $\mathrm{T}_{\mathrm{e}}$ gradient as supported by gyro-kinetic modelling. The experimentally observed ELM cycle is described qualitatively by the ideal PB model, as the stability boundary is moving closer to the operational point in $j-\alpha$ space with increasing pedestal width. A quantitative determination of the pedestal parameters at the ELM crash has not been possible with the ideal MHD modelling. This is not surprising if non-linear effects come into play, such as the coupling of MTMs located at the pedestal top with low-n ballooning modes in the pedestal.

In all experiments presented here the neoclassical electron collisionality [43] at the pedestal top is in the range of $v_{\text {e,neo }}{ }^{*} \sim 0.2-1.5$, i.e. one order of magnitude larger than expected in ITER plasmas. The possible particle pinch found in the post L-H transition transport studies as well as immediately after the ELM crash during the $\mathrm{n}_{\mathrm{e}}$ build up, which could be assigned to TEMs, might also occur at lower collisionalities. Such a pinch might help fuelling of ITER plasmas. The impurity flow profiles were studied in the Pfirsch-Schlüter, the plateau and the banana regime and could always be described by neoclassical theory [28]. Therefore, we assume that also in the ITER pedestal impurity ion transport will be neoclassical. Microtearing modes can have different driving mechanisms. They 
can exhibit growth rates, which decrease with decreasing collisionality [44], an effect that might be compensated, however, by lower $\mathrm{T}_{\mathrm{e}}$ gradient lengths or higher beta. If the drive is dominated by geometric effects [45, 46], collisionality will not change their appearance. In both cases MTMs might appear alongside ETG modes and KBMs in the ITER pedestal.

At ASDEX Upgrade these kinds of pedestal studies have improved our understanding of the processes which govern the pedestal transport. Future studies will address open questions such as the influence of impurities on the edge stability, the role of the divertor state for the fuelling efficiency and the change of inter-ELM pedestal dynamics with collisionality and shaping. Investigations of the ion heat transport channel in the pedestal are also planned. Further expansion of our diagnostic capabilities will be provided by a second ECEI system as well as multiple reflectometry systems at the LFS and the HFS.

\section{Acknowledgements:}

This work has been carried out within the framework of the EUROfusion Consortium and has received funding from the Euratom research and training programme 2014-2018 under grant agreement No 633053. The views and opinions expressed herein do not necessarily reflect those of the European Commission.

\section{References:}

[1] Viezzer et al., Nuclear Fusion 54, 1 (2014), 012003 Nucl. Fusion 53 (2013) 053005

[2] Pütterich et al., Nucl. Fusion 52 (2012) 083013

[3] Angioni et al., Plasma Phys. Control. Fusion 51 (2009) 124017

[4] Kinsey et al., Nucl. Fusion 51 (2011) 083001

[5] Diallo et al., Phys.Rev. Lett. 112 (2014) 115001

[6] Snyder et al., Nucl. Fusion 49 (2009) 085035

[7] Groebner et al., Nucl. Fusion 53 (2013) 093024

[8] Dickinson et al., Phys.Rev.Lett. 108 (2012) 135002

[9] Hatch et al, submitted to Nucl. Fusion

[10] Kurzan et al. Rev.Sci.Instr. 82 (2011) 103501

[11] Fischer et al., Plasma Phys. Control. Fusion 50 (2008) 085009

[12] Classen et al., Rev.Sci.Instr.81 (2010) 10D929

[13] Pütterich et al., EPS Conference on Plasma Physics, Sofia, Bulgaria, 2009, P1.158

[14] Rathgeber et al., Plasma Phys. Control. Fusion 55 (2013) 025004

[15] Viezzer et al., Rev.Sci.Instr.83 (2012) 103501 
[16] Dunne et al., Nucl. Fusion 52 (2012) 123014

[17] Willensdorfer et al., Plasma Phys. Control. Fusion 56 (2014) 025008 (10pp)

[12] Mlynek et al., Rev.Sci.Instr. 81 (2010) 033507

[19] Fischer et al., Fus.Sci.Techn.58 (2010) 675

[20] Neuhauser et al., Plasma Phys. Control. Fusion 44 (2002) 855

[21] McDermott et al., Plasma Phys. Control. Fusion 53.(2011) 124013

[22] Viezzer et al., Plasma Phys. Control. Fusion 55 (2013) 124037

[23] Connor, Plasma Phys. Control. Fusion 40 (1998) 531

[24] Kukushkin et al., Nucl. Fusion 43 (2003) 716

[25] Willensdorfer et al., Nucl. Fusion 53 (2013) 093020

[26] Willensdorfer et al., submitted to J.Nucl.Mat.

[27] Viezzer et al., Plasma Phys. Control. Fusion 56 (2014) 075018

[28] Viezzer et al, submitted to Nucl. Fusion

[29] Viezzer et al., Plasma Phys. Control. Fusion 55 (2013) 124037

[30] Fable et al., in preparation

[31] Peeters, Physics of Plasmas 7 (2000) 268

[32] Pütterich et al., J. Nucl. Mat. 415 (2011) S334

[33] Burckhart et al., Plasma Phys. Control. Fusion 52 (2010) 105010

[34] Connor, Plasma Phys. Control. Fusion 40 (1998) 191

[35] Snyder et al., Nucl. Fusion 49 (2009) 085035

[36] Miller et al., Physics of Plasmas 5 (1998) 973

[37] Konz et al., EPS Conference on Plasma Physics, Strasbourg, France, 2011, O2.103

[38] Burckhart, PHD thesis 'Different ELM regimes at ASDEX Upgrade and their linear stability analysis‘, LMU München, 2013.

[39] Jenko et al., Physics of Plasmas 7 (2000) 1904

[40] Told et al., Physics of Plasmas 15 (2008) 102306

[41] Schneider et al., Nucl. Fusion 53 (2013) 073039

[42] Manz et al., Plasma Phys. Control. Fusion 56 (2014) 035010

[43] Sauter et al., Physics of Plasmas 6 (1999) 2834

[44] Doerk et al., Physics of Plasmas 19 (2012) 055907

[45] Dickinson et al., Plasma Phys. Control. Fusion 55 (2013) 074006

[46] Saarelma et al., Nucl. Fusion 53 (2013) 123012 